\title{
Opioid agonist treatment and risk of mortality during opioid overdose public health emergency: population based retrospective cohort study
}

\author{
OPEN ACCESS
}

\author{
Lindsay A Pearce project coordinator ${ }^{1}$, Jeong Eun Min senior statistician ${ }^{1}$, Micah Piske project \\ coordinator ${ }^{1}$, Haoxuan Zhou statistician ${ }^{1}$, Fahmida Homayra statistician ${ }^{1}$, Amanda Slaunwhite \\ senior scientist ${ }^{2}$, Mike Irvine postdoctoral researcher ${ }^{2}$, Gina McGowan director of research \\ translation $^{3}$, Bohdan Nosyk research scientist and associate professor ${ }^{14}$
}

${ }^{1}$ Health Economic Research Unit, British Columbia Centre for Excellence in HIV/AIDS, Vancouver, BC, V6Z 1Y6, Canada; ${ }^{2}$ British Columbia Centre for Disease Control and Prevention, Vancouver, BC, V5Z 4R4, Canada; ${ }^{3}$ British Columbia Ministry of Mental Health and Addictions, Victoria, BC, V8W 9P1, Canada; ${ }^{4}$ Faculty of Health Sciences, Simon Fraser University, Burnaby, BC, V5A 1S6, Canada

\begin{abstract}
Abstract

Objective - To compare the risk of mortality among people with opioid use disorder on and off opioid agonist treatment (OAT) in a setting with a high prevalence of illicitly manufactured fentanyl and other potent synthetic opioids in the illicit drug supply.

Design - Population based retrospective cohort study.

Setting - Individual level linkage of five health administrative datasets capturing drug dispensations, hospital admissions, physician billing records, ambulatory care reports, and deaths in British Columbia, Canada.

Participants - 55347 people with opioid use disorder who received OAT between 1 January 1996 and 30 September 2018.

Main outcome measures - All cause and cause specific crude mortality rates (per 1000 person years) to determine absolute risk of mortality and all cause age and sex standardised mortality ratios to determine relative risk of mortality compared with the general population. Mortality risk was calculated according to treatment status (on OAT, off OAT), time since starting and stopping treatment (1, 2, 3-4, 5-12, >12 weeks), and medication type (methadone, buprenorphine/naloxone). Adjusted risk ratios compared the relative risk of mortality on and off OAT over time as fentanyl became more prevalent in the illicit drug supply.

Results $-7030(12.7 \%)$ of 55347 OAT recipients died during follow-up. The all cause standardised mortality ratio was substantially lower on OAT $(4.6,95 \%$ confidence interval 4.4 to 4.8$)$ than off OAT $(9.7,9.5$ to 10.0). In a period of increasing prevalence of fentanyl, the relative risk of mortality off OAT was 2.1 (95\% confidence interval 1.8 to 2.4 ) times higher than on OAT before the introduction of fentanyl, increasing to 3.4 ( 2.8 to 4.3 ) at the end of the study period ( $65 \%$ increase in relative risk).
\end{abstract}

Conclusions - Retention on OAT is associated with substantial reductions in the risk of mortality for people with opioid use disorder. The protective effect of OAT on mortality increased as fentanyl and other synthetic opioids became common in the illicit drug supply, whereas the risk of mortality remained high off OAT. As fentanyl becomes more widespread globally, these findings highlight the importance of interventions that improve retention on opioid agonist treatment and prevent recipients from stopping treatment.

\section{Introduction}

The global burden of disease due to opioid use disorder continues to grow. Between 1990 and 2016, the total number (age standardised prevalence) of people dependent on opioids increased from 18.2 million ( 360.8 per 100000 ) to 26.8 million (353.0 per 100000 ), resulting in 86200 deaths and 36.6 million years of life lost in 2016. ${ }^{1}$ In North America, the introduction of illicitly manufactured fentanyl and other highly potent, synthetic opioids in the drug supply has contributed to a rapidly worsening disease burden. ${ }^{2-7}$ In Canada, an unprecedented 4614 opioid related deaths occurred in 2018 , with nearly three quarters of these deaths involving fentanyl or other synthetic opioids. ${ }^{8}$ People with opioid use disorder are at high risk of exposure to these contaminants, ${ }^{9}$ which are up to 10000 times more potent than morphine. ${ }^{1011}$ This is contributing to a mounting global public health concern as fentanyl and other synthetic opioids become more widespread internationally. ${ }^{12}$

Opioid agonist treatment (OAT), primarily with methadone or buprenorphine/naloxone, is a safe and effective treatment for opioid use disorder that has been shown to suppress illicit opioid use and reduce the risk of death. ${ }^{13}{ }^{14} \mathrm{~A}$ recent systematic review 
and meta-analysis of observational studies conducted between 1974 and 2016 found that being on treatment was associated with a lower risk of death from all causes and from overdose. ${ }^{14}$ However, the risk of death changed according to time since starting or stopping treatment and differed by medication type. Pooled analyses concluded that all cause mortality was reduced by a factor of 3.2 (95\% confidence interval 2.7 to 3.9 ) when people were taking methadone and 2.2 (1.3 to 3.6 ) when they were taking buprenorphine/naloxone. The risk of death was highest in the first four weeks after stopping treatment (32.0 deaths per 100000 person years on methadone and buprenorphine/naloxone), with a swift decline in the risk of death observed thereafter. These findings are important because people frequently stop and start these medications, and this exposes them to repeated periods of high risk of death. Excess mortality after stopping treatment can be explained by a loss of tolerance to illicit opioids during treatment, ${ }^{15-20}$ which increases the risk of fatal overdose after resuming illicit opioid use. Other factors that may contribute to the high risk of death after stopping treatment include subsequent disruptions in care for concurrent conditions and lifestyle instability that increases the risk of death due to other causes. ${ }^{142021}$

The presence of more potent contaminants such as fentanyl in the illicit drug supply could increase these risks further. However, evidence examining the relation between opioid agonist treatment and mortality in settings with widespread availability of these contaminants in the illicit drug supply is limited. Therefore, our objective was to estimate the risk of mortality on and off opioid agonist treatment in a setting with a high prevalence of fentanyl and other potent synthetic opioids in the illicit drug supply.

\section{Methods}

\section{Data sources}

We did a retrospective cohort study based on linkage of five provincial health administrative databases to identify people accessing OAT in British Columbia, Canada, from 1 January 1996 to 30 September 2018. We used the PharmaNet database to identify OAT dispensations. PharmaNet covers all drugs distributed by pharmacies and hospital outpatient settings and includes details on type of drug dispensed (drug identification number), date of dispensation, and quantity of prescription. ${ }^{22}$ We used the Vital Statistics database to identify all deaths and their underlying cause during the follow-up period. Causes of death are initially determined by a physician, coroner, or medical examiner and subsequently abstracted by professionals and coded into the Vital Statistics database. This database captures all deaths registered in the province and includes a diagnostic code for the underlying cause of death and nature of injury. ${ }^{23}$ The Discharge Abstract Database captures all hospital admissions in the province and includes diagnostic codes for the most responsible diagnosis and up to 24 additional diagnostic codes for each admission. ${ }^{24}$ We primarily used this database to determine OAT treatment status during hospital admissions, as OAT administered in hospital is not included in the PharmaNet database. We used the Medical Services Plan and the National Ambulatory Care Reporting System databases to identify comorbidities related to opioid use disorder. The Medical Services Plan database captures all services provided by practitioners under the province's universal insurance programme and includes details on services administered, such as laboratory and diagnostic procedures. ${ }^{25}$ The National Ambulatory Care Reporting System database captures all levels of ambulatory care (emergency departments, day surgery, and medical and surgical day clinics within hospitals, the community, and private clinics) for 29 facilities in the province. ${ }^{26}$ The databases were deterministically linked using a unique, individual level personal health number. ${ }^{27}$

\section{Study population}

We identified all OAT recipients during the follow-up period on the basis of the presence of at least one OAT dispensation in PharmaNet (online appendix table A1). We followed all OAT recipients from the date of first OAT dispensation or prescription to either administrative loss to follow-up or death. As out-of-province migration is not explicitly captured in provincial health administrative datasets, we defined administrative loss to follow-up as no record of any kind in any of the linked databases for at least 66 months before the end of the follow-up period based on the empirical distribution of gap times in service delivery (appendix figure A1).

\section{Characteristics of OAT recipients}

We measured characteristics of OAT recipients at the time of first OAT dispensation during the follow-up period including age, sex, health authority (geographic healthcare delivery region) of residence, and calendar year. We determined the presence of opioid related comorbidities (non-alcohol and non-opioid substance use disorder, alcohol use disorder, mental health disorder, non-cancer chronic pain, hepatitis C virus, and HIV) on the basis of the presence of one or more hospital admission records, one or more ambulatory care records, at least three physician claims, or receipt of drug treatment for alcohol use disorder or hepatitis $\mathrm{C}$ virus during follow-up (appendix table A5).

\section{Key outcome measures Classification of deaths}

We extracted death records from the Vital Statistics database and categorised them as all cause, drug related (problematic drug use or accidental drug poisoning), other external cause (injury, poisoning, other consequences of external causes), infectious disease, other non-external cause, or unknown. ${ }^{28} \mathrm{We}$ also identified death records specific to opioid from the underlying cause of death and nature of injury code in the Vital Statistics database. We used ICD (international classification of diseases) diagnostic codes from the Discharge Abstract Database and the National Ambulatory Care Reporting System if the underlying cause of death from Vital Statistics was not available or the individual died in hospital. A list of diagnostic codes used to determine underlying cause of death is provided in appendix table A6.

\section{OAT exposure definition}

We defined exposure to OAT as any receipt of methadone, buprenorphine/naloxone, slow release oral morphine (available from June 2017), or injectable OAT (hydromorphone or diacetylmorphine; available from June 2014). We defined a period on OAT as a continuous period of dispensed medication with no interruptions in dispensed or prescribed doses lasting at least five days for methadone or slow release oral morphine, at least six days for buprenorphine/naloxone, and at least three days for injectable OAT, according to provincial prescribing guidelines. ${ }^{29}$ As OAT dispensations occurring in hospital are not recorded in the PharmaNet database, if hospital admissions occurred when patients were on OAT before admission we assumed that they continued treatment in hospital (appendix figure A2). 


\section{Attribution of deaths to periods on or off OAT}

We classified deaths occurring on a date on which OAT was dispensed or prescribed (in the case of take-home doses) or in hospital (for patients on OAT at the time of admission) as "on OAT." We classified deaths occurring at least one day after OAT was prescribed or dispensed or after discharge from a linked hospital admission as "off OAT."

\section{Attribution of deaths to time interval on and off OAT}

We evaluated the risk of mortality within one week, two weeks, three to four weeks, five to 12 weeks, and more than 12 weeks (up to 22.7 years) after patients started and stopped OAT, given the known risks of death immediately after stopping treatment. ${ }^{14}$ Although some recent studies have reported the risk of mortality in the first two weeks overall, ${ }^{15} 163031$ we present results separately in week one and week two to better characterise the differences in the risk of mortality after starting and stopping OAT. We chose a four week threshold to be consistent with a recent systematic review and meta-analysis and a 12 week threshold to coincide with eligibility guidelines for take-home dosing of methadone in British Columbia. ${ }^{1429}$

\section{Statistical analysis}

We used all cause and cause specific crude mortality rates to determine absolute risk of mortality and all cause age and sex standardised mortality ratios to determine the relative risk of mortality compared with the general population. To calculate crude mortality rates, we summed the number of deaths by category and calculated a rate per 1000 person years. We calculated person years from the first OAT dispensation or prescription (dating back to 1 January 1996) until death or end of follow-up (30 September 2018). We calculated crude rate ratios by dividing one crude mortality rate by the other. We calculated age and sex standardised mortality ratios by dividing the observed number of deaths in the cohort by the expected number of deaths based on the British Columbia population mortality rates by age group, sex, and calendar year. ${ }^{32} \mathrm{We}$ derived $95 \%$ confidence intervals from exact Poisson based intervals. $^{33}$

We calculated the risk of mortality according to medication type, treatment status (on OAT, off OAT), and time since starting and stopping treatment. We present these for methadone and buprenorphine/naloxone only, given the small number of people receiving injectable OAT and slow release oral morphine after these were made available in British Columbia in 2014 and 2017, respectively.

We then identified key dates of the opioid overdose public health emergency in British Columbia, including the date of the first death for which fentanyl was detected in the province (1 April 2012) and the date of the declaration of the provincial public health emergency (14 April 2016), as time indicators that reflected increasing fentanyl contamination. ${ }^{345}$ We calculated annual crude mortality rates and standardised mortality ratios for periods on OAT and off OAT. We used Poisson regression to calculate risk ratios to compare differences in the risk of mortality off OAT versus on OAT, before and after key dates, adjusting for age, sex, medication type (buprenorphine/naloxone only, methadone only), and time since starting or stopping treatment ( $\leq 4$ weeks, $>4$ weeks).

Finally, we did sensitivity analyses on the definition of death during OAT: strictly by the date of OAT dispensation or prescription without incorporating hospital admissions; and treating deaths occurring within the defined gap time for each medication as occurring on OAT. We used SAS 9.4 for all analyses.

\section{Patient and public involvement}

No patients were involved in setting the research question or the outcome measures, nor were they involved in developing plans for recruitment, design, or implementation of the study. No patients were asked to advise on interpretation or writing up of results. Local organisations of people who use drugs and people who have accessed opioid agonist treatment in Vancouver, Canada, will be engaged through an in-person meeting as part of the knowledge translation and dissemination process.

\section{Results \\ Characteristics of OAT recipients}

We identified 55347 OAT recipients in British Columbia between 1 January 1996 and 30 September 2018. At the time of first OAT dispensation or prescription, 35381 (63.9\%) had a mental health disorder, $30063(54.3 \%)$ had chronic pain, 35 $127(63.5 \%)$ had a concurrent substance use disorder, and 33 $691(60.9 \%)$ were between ages 25 and 44 (table 1); 3890 (7.0\%) were lost to follow-up.

\section{All cause mortality in overall sample}

We observed 7030 (12.7\%) deaths during follow-up. From 400 817 person years of follow-up, we observed an overall all cause crude mortality rate of 17.5 (95\% confidence interval 17.1 to 18.0) deaths per 1000 person years and a standardised mortality ratio of 7.2 (95\% confidence interval 7.1 to 7.4) (table 2). Standardised mortality ratios were highest among people under 20 years of age (15.0, 8.6 to 24.4), those with HIV (HIV positive 20.7, 19.4 to 22.0; HIV negative or unknown $6.5,6.4$ to 6.7 ), and those with hepatitis $\mathrm{C}$ virus (positive $16.5,15.8$ to 17.2; negative or unknown $6.0,5.8$ to 6.1 ).

\section{All cause mortality on and off OAT}

The risk of mortality was substantially lower during periods on OAT (standardised mortality ratio 4.6, 4.4 to 4.8) compared with periods off OAT (9.7, 9.5 to 10.0) (table 2). Standardised mortality ratios were 4.7 (4.5 to 4.9) and 9.5 (9.3 to 9.8) on and off OAT with methadone, respectively. Standardised mortality ratios were 2.9 (2.3 to 3.6$)$ and 11.3 (10.4 to 12.2) on and off OAT with buprenorphine/naloxone, respectively. Among 2197 deaths occurring on OAT, $425(19.3 \%)$ occurred on a date on which OAT was dispensed, $675(30.7 \%)$ occurred on a date on which OAT was prescribed (for take-home doses), and 1097 $(49.9 \%)$ occurred while the patient was in hospital.

\section{All cause mortality by time interval on and off OAT}

The risk of mortality was highest in the week after stopping treatment for both methadone and buprenorphine/naloxone. The most substantial differences in mortality were in the week after stopping treatment with methadone (standardised mortality ratio $84.4,78.0$ to 91.2 ), which exceeded the risk of mortality in the second week by a factor of 3.9 (week 2: 21.5, 17.6 to 26.0) (fig 1). The risk of mortality remained high during week two (standardised mortality ratio $21.5,17.6$ to 26.0 ), weeks three to four $(22.1,18.8$ to 25.9$)$, and weeks five to 12 (18.6, 16.8 to 20.5) after stopping treatment with methadone. A substantial decrease in the risk of mortality occurred after 12 weeks (standardised mortality ratio $7.3,7.0$ to 7.6 ). We observed a 
relatively higher risk of mortality in the first week (31.0, 23.4 to 40.2) compared with later weeks after stopping treatment with buprenorphine/naloxone. The risk of mortality was lower after stopping treatment with buprenorphine/naloxone compared with methadone, but not consistently so overall (rate ratio 1.0, 0.9 to $1.1 ; \mathrm{P}=0.82$ ). In the week immediately after stopping OAT, however, the risk of mortality was 2.6 (1.9 to 3.4$)$ times higher for methadone than for buprenorphine/naloxone. Appendix table A7 provides all mortality rates by time since starting and stopping OAT and medication type.

\section{All cause mortality on and off OAT in presence of fentanyl}

Figure 2 shows all cause standardised mortality ratios on and off OAT from 2010 to 2018. Between 2010 and 2018, we observed substantial increases in the risk of mortality off OAT, whereas the risk of mortality on OAT remained stable after the first death from overdose in which fentanyl was detected (01 April 2012) and the declaration of the provincial public health emergency on opioid overdose (14 April 2016). Appendix table A8 provides annual crude mortality rates and standardised mortality ratios underlying the figure.

Compared with periods on OAT, the adjusted relative risk of mortality off OAT was 2.1 (1.8 to 2.4) times higher before the introduction of fentanyl, increased to 2.6 (2.1 to 3.2) after the introduction of fentanyl in the illicit drug supply and before the declaration of the public health emergency ( $26 \%$ increase), and further increased to 3.4 (2.8 to 4.3) after the declaration of the public health emergency (65\% increase from pre-fentanyl period) (table 3).

\section{Cause specific mortality}

The drug related crude mortality rate was 5.4 (5.2 to 5.7) deaths per 1000 person years (table 2). Drug related mortality was lower on OAT (2.1 (1.9 to 2.3) deaths per 1000 person years) relative to periods off OAT (8.8 (8.4 to 9.3) deaths per 1000 person years). The risk of drug related mortality was highest in the week after stopping OAT with methadone (55.3 (48.5 to $62.8)$ deaths per 1000 person years) and starting OAT with methadone (7.0 (4.7 to 9.9) deaths per 1000 person years) and gradually decreased over time (fig 3). We observed elevated drug related mortality rates in the first week (3.3 (0.7 to 9.5) deaths per 1000 person years) and second week (4.2 (0.9 to 12.3) deaths per 1000 person years) after starting buprenorphine/naloxone and in the first week (23.3 (14.6 to 35.3) deaths per 1000 person years) after stopping buprenorphine/naloxone. The opioid related crude mortality rate was 3.5 (3.3 to 3.7) deaths per 1000 person years, with a similar pattern to the drug related mortality rate by time since starting and stopping treatment and by medication type (appendix table A7).

\section{Sensitivity analysis}

Sensitivity analyses on alternate definitions of OAT episodes did not alter the conclusions on the protective effects of OAT against mortality (appendix table A9). Compared with the baseline definition (risk ratio off OAT $v$ on OAT 2.5, 2.1 to 2.9), periods on OAT constructed strictly by the OAT dispensation records and excluding hospital admissions estimated a higher protective effect of OAT on mortality risk (6.2, 4.9 to 7.7). However, defining deaths during the indicated gap in dispensed or prescribed doses for each medication $(<5$ days for methadone or slow release oral morphine, $<6$ days for buprenorphine/naloxone, and $<3$ days for injectable OAT) as occurring "on OAT" resulted in more conservative estimates of the reduction in mortality (risk ratio 1.6, 1.4 to 1.7) compared with defining deaths during this gap as occurring "off OAT" $(2.5,2.1$ to 2.9$)$.

\section{Discussion}

In a population based cohort of more than 55000 treatment recipients from 1 January 1996 to 30 September 2018, OAT had a clear protective effect on the risk of mortality that strengthened after the introduction of fentanyl in the illicit drug supply. The risk of mortality in patients on OAT did not increase as illicit opioids increased in potency. Our key finding was that the relative risk of mortality in patients off OAT was $2.1(95 \%$ confidence interval 1.8 to 2.4) times higher than in those on OAT before the introduction of fentanyl, increasing to 3.4 (2.8 to 4.3$)$ at the end of the study period $(65 \%$ increase in relative risk). The risk of all cause and drug related mortality was substantially lower in patients on OAT than off OAT. We also observed dramatic increases in the risk of mortality immediately after patients stopped OAT. The risk of mortality after starting and stopping treatment was consistently lower among patients on buprenorphine/naloxone than on methadone.

\section{Comparison with other studies}

The association between OAT and mortality has been extensively studied; however, this study uniquely examines this relation in a setting with a high prevalence of fentanyl and other potent synthetic opioids in the illicit drug supply. Our finding that OAT was associated with an even greater relative risk reduction after the emergence of fentanyl is unprecedented to date. A recent study in Massachusetts, a jurisdiction with a similarly high prevalence of fentanyl in the illicit drug supply, found that OAT was associated with substantial reductions in mortality among people engaging in treatment after a non-fatal overdose. $^{36}$

Our findings of a substantially lower risk of mortality on OAT compared with off OAT are consistent with those reported in other jurisdictions. A 2009 study of more than 40000 treatment recipients in Australia reported a standardised mortality ratio of 4.5 (4.3 to 4.8) on OAT and 8.0 (7.7 to 8.3) off OAT. ${ }^{15}$ These findings are nearly the same as those observed in British Columbia. A 2017 systematic review and meta-analysis reported a pooled all cause mortality rate of 11.3 and 36.1 per 1000 person years on and off methadone and 4.3 and 9.5 per 1000 person years on and off buprenorphine/naloxone, representing a relative risk reduction of 3.2 (2.7 to 3.9) on methadone and 2.2 (1.3 to 3.6) on buprenorphine/naloxone. ${ }^{14}$ These pooled mortality rates are slightly different from those observed in our study (11.1 and 24.3 per 1000 person years on and off methadone and 6.6 and 24.0 per 1000 person years on and off buprenorphine/naloxone). This may reflect differences in the characteristics of people accessing each form of treatment, the total person years of follow-up, healthcare settings in which OAT is delivered, and clinical prescribing guidelines.

Differences between this study and others in the risk of mortality in patients on buprenorphine/naloxone could reflect a relatively short period of follow-up time on this medication. ${ }^{163738}$ Furthermore, all existing studies examining mortality among people treated with buprenorphine/naloxone were conducted in Australia. Pooled trends analysis involving previous studies showed an increased risk of mortality in the first four weeks after stopping treatment with both methadone and buprenorphine/naloxone, ${ }^{14}$ which was also observed in British Columbia. We observed a moderately higher mortality risk 
during this period, which may be attributable to the high prevalence of fentanyl in the illicit drug supply during the follow-up period.

The risk of mortality after starting and stopping treatment was consistently lower for buprenorphine/naloxone than for methadone. This is consistent with previous systematic reviews of observational studies and clinical trials that showed a superior safety profile of buprenorphine/naloxone relative to methadone. ${ }^{1339-42}$ As a partial agonist, the effects of buprenorphine/naloxone plateau at higher doses, contributing to a reduced risk of overdose and respiratory depression after the start of treatment compared with methadone..$^{29} 434$ Buprenorphine/naloxone has a slower dissociation rate from $\mu$ opioid receptors, resulting in a longer duration of action, prolonged suppression of opioid withdrawal, and a brief reduction in the effects of exogenous opioids, which may contribute to a lower risk of death after stopping treatment. ${ }^{41} 4345$

Finally, differences in the methods used to construct OAT episodes from health administrative data are evident in the existing literature. We treated deaths that occurred during the defined gap time for each medication as occurring out of treatment, which is in agreement with two recent Canadian studies ${ }^{28}{ }^{30}$ but in contrast to others that treated these deaths as occurring during treatment. ${ }^{153138}$ Sensitivity analysis on the latter definition showed conservative estimates of mortality reduction during periods on OAT. Additionally, our decision to incorporate hospital admissions into OAT episodes resulted in higher mortality rates during OAT than we would otherwise have observed.

\section{Strengths and limitations of study}

This population based study represents one of the largest cohorts of OAT recipients to date, overcoming previously cited limitations that prevented a more granular analysis of mortality risk according to treatment status. ${ }^{146} \mathrm{We}$ present findings that confirm results of previous systematic reviews and meta-analyses, ${ }^{147}$ but they also contribute novel findings by direct examination of this relation after fentanyl became prevalent in the illicit drug supply.

We note several limitations. Firstly, the PharmaNet data extract excluded OAT administered in hospital and to federally insured people, including Royal Canadian Mounted Police, Canadian Forces personnel, and veterans. The Medical Services Plan database may exclude some services delivered by physicians who were funded through an alternative payment method (contracted service, sessional, and salaried), as these physicians are paid whether or not they submit a billing claim. Also, these datasets exclude healthcare services and deaths outside of British Columbia. Therefore, misclassification was possible if a patient received OAT before moving into the province and died shortly thereafter or if a person died outside of the province.

Secondly, we were unable to distinguish cause specific deaths in the Vital Statistics dataset in later years. In total, the underlying cause for 690 (9.8\%) of the 7030 deaths was "unknown;" $56.5 \%$ of deaths in 2018 and $12.6 \%$ of deaths in 2017 were still under investigation at the time of data extraction and categorised as "unknown" in the Vital Statistics database. Therefore, a higher proportion of deaths on buprenorphine/naloxone were classified as unknown relative to methadone, limiting inferences on cause specific mortality across treatment modalities.

Finally, this study reports findings from a descriptive analysis and should be interpreted accordingly. Patients were not randomised to treatment status or to the specific treatment modalities examined. As a result, underlying differences may exist in patient populations, accounting to some degree for observed differences in the risk of mortality across strata. Sordo et al note the potential for confounding in comparisons of mortality risk on and off OAT in observational research. ${ }^{14}$ Observed differences in mortality risk may be partially attributable to characteristics other than treatment status, such as severity of opioid use disorder, mental and physical health status, and lifestyle stability, which fluctuate over time and affect the decision to start and stop treatment. ${ }^{14}{ }^{15}$ Recent studies controlling for characteristics of patients and treatment at baseline concluded that adjustment had no significant effect on relative mortality risk or even increased relative risks comparing mortality off and on OAT. ${ }^{14}$ In this study, we did not observe significant differences in measures of relative mortality risk after accounting for patients' characteristics. Additionally, differences in treatment delivery and pharmacological properties of specific OAT medications should be considered when interpreting study findings. This study was conducted in a universal healthcare setting, where nearly all healthcare is provided free of charge and all OAT medications are available in office based settings, which differs substantially from treatment delivery in other jurisdictions such as the US. Nevertheless, the consistency of our findings with the multitude of observational studies and randomised clinical trials in this field is reassuring. Further analyses accounting for possible time dependent confounding are needed to obtain an unbiased estimate of the causal effect of OAT on mortality and represent an important future investigation using these data. ${ }^{48}$

\section{Conclusions and policy implications}

Despite advances in opioid agonist treatment and a growing body of evidence showing the protective effects of engagement and retention on these medications, ${ }^{147}$ mortality among people with opioid use disorder continues to increase in North America. ${ }^{49}$ This study provides strong evidence that OAT is an effective intervention to reduce the risk of mortality in the presence of illicitly manufactured fentanyl and other potent synthetic opioids. In a large population of OAT recipients, we observed a clear protective effect of OAT retention on mortality risk that strengthened during an opioid overdose public health emergency. As fentanyl becomes more widespread globally, ${ }^{12}$ interventions that improve treatment retention and identify and re-engage people at high risk of stopping treatment are more important than ever.

\section{What is already known on this topic}

Opioid agonist treatment is a safe and effective treatment for opioid use disorder that reduces the risk of all cause and overdose mortality

People with opioid use disorder commonly stop and start opioid agonist treatment, and their risk of death increases immediately after stopping

The prevalence of illicitly manufactured fentanyl and other highly potent synthetic opioids has increased in the illicit drug supply, increasing the risk of mortality among people with opioid use disorde

\section{What this study adds}

The risk of all cause mortality was substantially lower while patients were on OAT than when they were off OAT

The risk of mortality on OAT did not increase as fentanyl and other potent synthetic opioids were introduced into the illicit drug supply, resulting in stronger protective benefits of treatment after this 
and review. BN, JM, MP, AS, MI, and GM provided critical revisions to the manuscript. BN had the idea for the study and secured funding with the support of LAP and JM. All authors approved the final draft. The corresponding author attests that all listed authors meet authorship criteria and that no others meeting the criteria have been omitted. BN is the guarantor.

Funding: By contribution agreement 1819-HQ-000036 from Health Canada Substance Use and Addictions Program (SUAP). The funding source was independent of the design of this study and did not have any role during its execution, analyses, data interpretation, writing, or decision to submit results. All authors had full access to the results in the study and take responsibility for the integrity of the data and accuracy of analysis.

Competing interests: All authors have completed the ICMJE uniform disclosure form at www.icmje.org/coi_disclosure.pdf and declare: funding support from Health Canada SUAP; no financial relationships with any organisations that might have had an interest in the submitted work in the previous three years; no relationships or activities that could appear to have influenced the submitted work.

Ethical approval: This study was mandated by the British Columbia Ministry of Mental Health and Addictions as part of the response to the provincial opioid overdose public health emergency. The Providence Health Care Research Institute and the Simon Fraser University Office of Research Ethics determined it to be exempt from research ethics board review as per Article 2.5 of the Tri-Council Policy Statement: Ethical Conduct for Research Involving Humans.

Data sharing: Study protocol and statistical code are available from the corresponding author (bnosyk@cfenet.ubc.ca). All inferences, opinions, and conclusions drawn in this manuscript do not reflect the opinions or policies of the British Columbia Ministry of Health or the data steward(s).

Transparency: The lead author affirms that the manuscript is an honest, accurate, and transparent account of the study being reported; that no important aspects of the study have been omitted; and that any discrepancies from the study as originally planned (and, if relevant, registered) have been explained.

Dissemination to participants and related patient and public communities: Key findings will be disseminated to local organisations of people who use drugs and people who have accessed opioid agonist treatment during an in-person meeting in Vancouver, Canada.

1 Degenhardt L, Charlson F, Ferrari A, etal. GBD 2016 Alcohol and Drug Use Collaborators. The global burden of disease attributable to alcohol and drug use in 195 countries and territories, 1990-2016: a systematic analysis for the Global Burden of Disease Study 2016. Lancet Psychiatry 2018;5:987-1012. 10.1016/S2215-0366(18)30337-7 30392731

2 Belzak L, Halverson J. The opioid crisis in Canada: a national perspective. Health Promot Chronic Dis Prev Can 2018;38:224-33. 10.24095/hpcdp.38.6.02 29911818

3 British Columbia Coroners Service. Fentanyl-detected illicit drug overdose deaths: January 1, 2012 to January 31, 2019. 2019. https://www2.gov.bc.ca/assets/gov/birth-adoptiondeath-marriage-and-divorce/deaths/coroners-service/statistical/fentanyl-detected-overdose. pdf.

4 Zoorob M. Fentanyl shock: The changing geography of overdose in the United States. Int J Drug Policy 2019;70:40-6. 10.1016/j.drugpo.2019.04.010 31079029

5 Gomes T, Tadrous M, Mamdani MM, Paterson JM, Juurlink DN. The burden of opioid-related mortality in the United States. JAMA Netw Open 2018;1:e180217. 10.1001/jamanetworkopen.2018.021730646062

6 The Lancet Public Health. A public health approach to Canada's opioid crisis. Lancet Public Health 2018:3:e204. 10.1016/S2468-2667(18)30078-1 29709648

7 Ye X, Sutherland J, Henry B, Tyndall M, Kendall PRW. At-a-glance - Impact of drug overdose-related deaths on life expectancy at birth in British Columbia. Health Promot Chronic Dis Prev Can 2018;38:248-51. 10.24095/hpcdp.38.6.05 29911821

8 Government of Canada. Opioid-related harms in Canada. 2020. https://health-infobase. canada.ca/substance-related-harms/opioids.

9 Ochalek TA, Parker MA, Higgins ST, Sigmon SC. Fentanyl exposure among patients seeking opioid treatment. J Subst Abuse Treat 2019;96:23-5. 10.1016/j.jsat.2018.10.007 30466544

10 Fairbairn N, Coffin PO, Walley AY. Naloxone for heroin, prescription opioid, and illicitly made fentanyl overdoses: Challenges and innovations responding to a dynamic epidemic. Int J Drug Policy 2017;46:172-9. 10.1016/j.drugpo.2017.06.005 28687187

11 Misailidi N, Papoutsis I, Nikolaou P, Dona A, Spiliopoulou C, Athanaselis S. Fentanyls continue to replace heroin in the drug arena: the cases of ocfentanil and carfentanil. Forensic Toxicol 2018;36:12-32. 10.1007/s11419-017-0379-4 29367860

12 United Nations Office on Drugs and Crime. World Drug Report. 2019. https://wdr.unodc. org/wdr2019.

13 Mattick RP, Breen C, Kimber J, Davoli M. Buprenorphine maintenance versus placebo or methadone maintenance for opioid dependence. Cochrane Database Syst Rev 2014;(2):CD002207. 10.1002/14651858.CD002207.pub4 24500948

14 Sordo L, Barrio G, Bravo MJ, etal . Mortality risk during and after opioid substitution treatment: systematic review and meta-analysis of cohort studies. BMJ 2017;357:j1550. 10.1136/bmj.j1550 28446428

15 Degenhardt L, Randall D, Hall W, Law M, Butler T, Burns L. Mortality among clients of a state-wide opioid pharmacotherapy program over 20 years: risk factors and lives saved. Drug Alcohol Depend 2009;105:9-15. 10.1016/j.drugalcdep.2009.05.021 19608355
16 Cornish R, Macleod J, Strang J, Vickerman P, Hickman M. Risk of death during and after opiate substitution treatment in primary care: prospective observational study in UK General Practice Research Database. BMJ 2010;341:c5475. 10.1136/bmj.c5475 20978062

17 Davoli M, Bargagli AM, Perucci CA, etal. VEdeTTE Study Group. Risk of fatal overdose during and after specialist drug treatment: the VEdeTTE study, a national multi-site prospective cohort study. Addiction 2007;102:1954-9.

10.1111/j.1360-0443.2007.02025.x 18031430

18 Cousins G, Teljeur C, Motterlini N, McCowan C, Dimitrov BD, Fahey T. Risk of drug-related mortality during periods of transition in methadone maintenance treatment: a cohort study. $J$ Subst Abuse Treat 2011;41:252-60. 10.1016/j.jsat.2011.05.001 21696913

19 White JM, Irvine RJ. Mechanisms of fatal opioid overdose. Addiction 1999;94:961-72. 10.1046/j.1360-0443.1999.9479612.x 10707430

20 Buster MC, van Brussel GH, van den Brink W. An increase in overdose mortality during the first 2 weeks after entering or re-entering methadone treatment in Amsterdam. Addiction 2002;97:993-1001. 10.1046/.1360-0443.2002.00179.x 12144602

21 McCowan C, Kidd B, Fahey T. Factors associated with mortality in Scottish patients receiving methadone in primary care: retrospective cohort study. BMJ 2009;338:b2225. 10.1136/bmj.b2225 19535400

22 British Columbia Ministry of Health. PharmaNet. British Columbia Ministry of Health, 2019 https://www2.gov.bc.ca/gov/content/health/health-drug-coverage/pharmacare-for-bcresidents/pharmanet.

23 British Columbia Vital Statistics Agency. Vital statistics deaths. British Columbia Ministry of Health, 2019. https://www2.gov.bc.ca/gov/content/life-events.

24 British Columbia Ministry of Health. Discharge abstract database (hospital separations). British Columbia Ministry of Health, 2019. https://www.popdata.bc.ca/data/health/dad.

25 British Columbia Ministry of Health. Medical Services Plan (MSP). British Columbia Ministry of Health, 2019. https://www2.gov.bc.ca/gov/content/health/health-drug-coverage/msp.

26 British Columbia Ministry of Health. National Ambulatory Care Reporting System (NACRS). British Columbia Ministry of Health, 2019. https://www.popdata.bc.ca/data/health/nacrs.

27 Government of British Columbia. Personal health identification. 2019. https://www2.gov. bc.ca/gov/content/health/health-drug-coverage/msp/bc-residents/personal-healthidentification.

28 Russolillo A, Moniruzzaman A, Somers JM. Methadone maintenance treatment and mortality in people with criminal convictions: A population-based retrospective cohort study from Canada. PLoS Med 2018;15:e1002625. 10.1371/journal.pmed.1002625 30063699

29 Government of British Columbia. A guideline for the clinical management of opioid use disorder. 2017. https://www2.gov.bc.ca/assets/gov/health/practitioner-pro/bc-guidelines/ bc_oud guidelines.pdf.

30 Evans E, Li L, Min J, etal . Mortality among individuals accessing pharmacological treatment for opioid dependence in California, 2006-10. Addiction 2015;110:996-1005. 10.1111/add. 1286325644938

31 Cousins G, Boland F, Courtney B, Barry J, Lyons S, Fahey T. Risk of mortality on and off methadone substitution treatment in primary care: a national cohort study. Addiction 2016:111:73-82. 10.1111/add.13087 26234389

32 Statistics Canada. Deaths and mortality rates, by age group. 2019. https://www150.statcan. gc.ca/t1/tbl1/en/tv.action?pid=1310071001

33 Ulm K. A simple method to calculate the confidence interval of a standardized mortality ratio (SMR). Am J Epidemiol 1990;131:373-5. 10.1093/oxfordjournals.aje.a115507 2296988

34 British Columbia Coroners Service. Illicit drug overdose deaths in BC: January 1, 2009 to October 31, 2019. 2019. https://www2.gov.bc.ca/assets/gov/birth-adoption-deathmarriage-and-divorce/deaths/coroners-service/statistical/illicit-drug.pdf.

35 Government of British Columbia. Provincial health officer declares public health emergency. 2016. https://news.gov.bc.ca/releases/2016HLTH0026-000568.

36 Larochelle MR, Bernson D, Land T, etal . Medication for opioid use disorder after nonfatal opioid overdose and association with mortality: a cohort study. Ann Intern Med 2018:169:137-45. 10.7326/M17-3107 29913516

37 Reece AS. Favorable mortality profile of naltrexone implants for opiate addiction. $J$ Addict Dis 2010;29:30-50. 10.1080/10550880903435988 20390697

38 Kimber J, Larney S, Hickman M, Randall D, Degenhardt L. Mortality risk of opioid substitution therapy with methadone versus buprenorphine: a retrospective cohort study. Lancet Psychiatry 2015;2:901-8. 10.1016/S2215-0366(15)00366-1 26384619

39 Marteau $\mathrm{D}, \mathrm{McD}$ onald $\mathrm{R}$, Patel $\mathrm{K}$. The relative risk of fatal poisoning by methadone or buprenorphine within the wider population of England and Wales. BMJ Open 2015;5:e007629.26024998

40 Chou R, Weimer MB, Dana T. Methadone overdose and cardiac arrhythmia potential: findings from a review of the evidence for an American Pain Society and College on Problems of Drug Dependence clinical practice guideline. J Pain 2014;15:338-65. 10.1016/j.jpain.2014.01.495 24685459

41 Davids E, Gastpar M. Buprenorphine in the treatment of opioid dependence. Eur Neuropsychopharmacol 2004;14:209-16. 10.1016/S0924-977X(03)00146-9 15056480

42 Maremmani I, Gerra G. Buprenorphine-based regimens and methadone for the medical management of opioid dependence: selecting the appropriate drug for treatment. $A m \mathrm{~J}$ Addict 2010;19:557-68. 10.1111/j.1521-0391.2010.00086.x 20958853

43 Sporer KA. Buprenorphine: a primer for emergency physicians. Ann Emerg Med 2004:43:580-4. 10.1016/j.annemergmed.2003.11.006 15111917

44 Tetrault JM, Fiellin DA. Current and potential pharmacological treatment options for maintenance therapy in opioid-dependent individuals. Drugs 2012;72:217-28. 10.2165/11597520-000000000-00000 22235870

45 Srivastava A, Kahan M. Buprenorphine: a potential new treatment option for opioid dependence. CMAJ 2006;174:1835-6. 10.1503/cmaj.050658 16785455

46 Manhapra A, Rosenheck R, Fiellin DA. Opioid substitution treatment is linked to reduced risk of death in opioid use disorder. BMJ 2017;357:j1947. 10.1136/bmj.j1947 28446438

47 Degenhardt L, Bucello C, Mathers B, etal . Mortality among regular or dependent users of heroin and other opioids: a systematic review and meta-analysis of cohort studies. Addiction 2011;106:32-51. 10.1111/j.1360-0443.2010.03140.x 21054613

48 Robins J, Hernan M. Estimation of the causal effects of time varying exposures. Chapman \& Hall, 2009.

49 Gostin LO, Hodge JGJJr, Noe SA. Reframing the opioid epidemic as a national emergency. JAMA 2017;318:1539-40. 10.1001/jama.2017.13358 28832871

\section{Accepted: 18022020}

(c) Author(s) (or their employer(s)) 2019. Re-use permitted under CC BY-NC. No commercial re-use. See rights and permissions. Published by 
BMJ.http://creativecommons.org/licenses/by-nc/4.0/This is an Open Access article distributed in accordance with the Creative Commons Attribution Non Commercial (CC BY-NC 4.0) license, which permits others to distribute, remix, adapt, build upon this work non-commercially, and license their derivative works on different terms, provided the original work is properly cited and the use is non-commercial. See: http://creativecommons. org/licenses/by-nc/4.0/. 


\section{Tables}

Table 1| Baseline characteristics of recipients of opioid agonist treatment (OAT) in British Columbia, Canada, 1 January 1996 to 30 September 2018

\begin{tabular}{|c|c|}
\hline Characteristic & No (\%) \\
\hline OAT recipients & $55347(100)$ \\
\hline \multicolumn{2}{|l|}{ Age group*: } \\
\hline$<20$ years & $2210(4.0)$ \\
\hline 20-24 years & $8302(15.0)$ \\
\hline 25-34 years & $19566(35.4)$ \\
\hline $35-44$ years & $14125(25.5)$ \\
\hline $45-54$ years & $7508(13.6)$ \\
\hline$>54$ years & $3628(6.6)$ \\
\hline Female sex & $18879 / 55339(34.1)$ \\
\hline Health authority $\dagger$ of residence*: & $(n=53982)$ \\
\hline Interior & $8725(16.2)$ \\
\hline Fraser & $19464(36.1)$ \\
\hline Vancouver Coastal & $14122(26.2)$ \\
\hline Vancouver Island & $9408(17.4)$ \\
\hline Northern & $2263(4.2)$ \\
\hline \multicolumn{2}{|c|}{ Comorbidity related to opioid use disorder ${ }^{\star} \ddagger$ : } \\
\hline Substance use disorder§ & $35127(63.5)$ \\
\hline Alcohol use disorder & $10786(19.5)$ \\
\hline Mental ill health & $35381(63.9)$ \\
\hline Chronic pain & $30063(54.3)$ \\
\hline Hepatitis $C$ virus & $1472(2.7)$ \\
\hline HIV & $1267(2.3)$ \\
\hline \multicolumn{2}{|l|}{ Calendar year*: } \\
\hline $1996-2000$ & $9982(18.0)$ \\
\hline $2001-06$ & $8064(14.6)$ \\
\hline 2007-12 & $13627(24.6)$ \\
\hline 2013-18 & $23674(42.8)$ \\
\hline
\end{tabular}

${ }^{*}$ At first OAT dispensation.

† Geographic healthcare delivery region.

$¥$ See appendix table A5 for ICD-9/ICD-10 codes used to identify concurrent chronic conditions.

$\S$ Excludes opioid use disorder and alcohol use disorder. 


\begin{tabular}{|c|c|c|c|c|c|c|}
\hline \multirow[b]{2}{*}{ Characteristic } & \multirow{2}{*}{$\begin{array}{l}\text { Person years of } \\
\text { follow-up }\end{array}$} & \multicolumn{3}{|c|}{ All cause } & \multicolumn{2}{|c|}{ Drug related $^{*}$} \\
\hline & & No of deaths & CMR† (95\% Cl) & SMR $(95 \% \mathrm{Cl})$ & No of deaths & CMR† $(95 \% \mathrm{Cl})$ \\
\hline Overall & 400817 & 7030 & $17.5(17.1$ to 18.0$)$ & $7.2(7.1$ to 7.4$)$ & 2184 & 5.4 (5.2 to 5.7$)$ \\
\hline Female sex & 142864 & 2274 & 15.9 (15.3 to 16.6$)$ & 9.9 (9.5 to 10.3$)$ & 668 & 4.7 (4.3 to 5.0$)$ \\
\hline Male sex & 257906 & 4756 & $18.4(17.9$ to 19.0$)$ & 6.4 (6.2 to 6.6$)$ & 1516 & 5.9 (5.6 to 6.2$)$ \\
\hline \multicolumn{7}{|l|}{ OAT status: } \\
\hline On OAT & 202315 & 2197 & 10.9 (10.4 to 11.3$)$ & $4.6(4.4$ to 4.8$)$ & 429 & 2.1 (1.9 to 2.3 ) \\
\hline Buprenorphine/naloxone & 13190 & 87 & $6.6(5.3$ to 8.1$)$ & $2.9(2.3$ to 3.6$)$ & 26 & $2.0(1.3$ to 2.9$)$ \\
\hline Methadone & 188113 & 2085 & 11.1 (10.6 to 11.6$)$ & $4.7(4.5$ to 4.9$)$ & 398 & $2.1(1.9$ to 2.3$)$ \\
\hline Off OAT & 198502 & 4833 & $24.3(23.7$ to 25.0$)$ & 9.7 (9.5 to 10.0$)$ & 1755 & $8.8(8.4$ to 9.3$)$ \\
\hline Buprenorphine/naloxone & 23712 & 570 & $24.0(22.1$ to 26.1$)$ & 11.3 (10.4 to 12.2$)$ & 269 & 11.3 (10.0 to 12.8 \\
\hline Methadone & 174431 & 4237 & 24.3 (23.6 to 25.0 ) & 9.5 (9.3 to 9.8 ) & 1482 & 8.5 (8.1 to 8.9$)$ \\
\hline \multicolumn{7}{|l|}{ Age group: } \\
\hline$<20$ years & $x$ & $x$ & $5.3(3.0$ to 8.7$)$ & 15.0 (8.6 to 24.4 ) & $x$ & $3.0(1.4$ to 5.7$)$ \\
\hline 20-24 years & 22579 & 187 & 8.3 (7.1 to 9.6$)$ & 13.1 (11.3 to 15.1$)$ & 106 & 4.7 (3.8 to 5.7$)$ \\
\hline 25-34 years & 107219 & 986 & 9.2 (8.6 to 9.8$)$ & $10.8(10.2$ to 11.5$)$ & 495 & $4.6(4.2$ to 5.0$)$ \\
\hline $35-44$ years & 122003 & 1510 & $12.4(11.8$ to 13.0$)$ & $9.5(9.0$ to 10.0$)$ & 646 & $5.3(4.9$ to 5.7$)$ \\
\hline $45-54$ years & 95300 & 1981 & 20.8 (19.9 to 21.7$)$ & 7.5 (7.2 to 7.9$)$ & 620 & $6.5(6.0$ to 7.0$)$ \\
\hline$>54$ years & 50670 & 2350 & 46.4 (44.5 to 48.3$)$ & $5.3(5.1$ to 5.5$)$ & 308 & 6.1 (5.4 to 6.8$)$ \\
\hline \multicolumn{7}{|l|}{ Alcohol use disorderł: } \\
\hline No & 293181 & 4175 & $14.2(13.8$ to 14.7$)$ & $5.8(5.7$ to 6.0$)$ & 1293 & $4.4(4.2$ to 4.7$)$ \\
\hline Yes & 107636 & 2855 & 26.5 (25.6 to 27.5$)$ & $11.1(10.7$ to 11.5$)$ & 891 & $8.3(7.7$ to 8.8$)$ \\
\hline \multicolumn{7}{|l|}{ Substance use disorder§: } \\
\hline No & 68692 & 889 & $12.9(12.1$ to 13.8$)$ & 3.8 (3.6 to 4.1$)$ & 48 & $0.7(0.5$ to 0.9$)$ \\
\hline Yes & 332125 & 6141 & $18.5(18.0$ to 19.0$)$ & 8.3 (8.1 to 8.5$)$ & 2136 & $6.4(6.2$ to 6.7$)$ \\
\hline \multicolumn{7}{|l|}{ Mental ill healthף: } \\
\hline No & 75448 & 1240 & $16.4(15.5$ to 17.4$)$ & $6.5(6.2$ to 6.9$)$ & 332 & $4.4(3.9$ to 4.9$)$ \\
\hline Yes & 325369 & 5790 & 17.8 (17.3 to 18.3$)$ & 7.4 (7.2 to 7.6$)$ & 1852 & $5.7(5.4$ to 6.0$)$ \\
\hline \multicolumn{7}{|l|}{ Chronic pain ${ }^{\star *}$ : } \\
\hline No & 122329 & 1796 & $14.7(14.0$ to 15.4$)$ & $7.6(7.2$ to 7.9$)$ & 597 & $4.9(4.5$ to 5.3$)$ \\
\hline Yes & 278489 & 5234 & 18.8 (18.3 to 19.3$)$ & 7.1 (6.9 to 7.3$)$ & 1587 & $5.7(5.4$ to 6.0$)$ \\
\hline \multicolumn{7}{|l|}{ Hepatitis C virus: } \\
\hline Unknown/not† & 366602 & 5098 & 13.9 (13.5 to 14.3$)$ & $6.0(5.8$ to 6.1$)$ & 1824 & $5.0(4.7$ to 5.2$)$ \\
\hline Yes & 34215 & 1932 & 56.5 (54.0 to 59.0$)$ & $16.5(15.8$ to 17.2$)$ & 360 & 10.5 (9.5 to 11.7$)$ \\
\hline \multicolumn{7}{|l|}{ HIV: } \\
\hline Unknown/not† & 378852 & 6043 & $16.0(15.6$ to 16.4$)$ & $6.5(6.4$ to 6.7$)$ & 1933 & $5.1(4.9$ to 5.3$)$ \\
\hline Yes & 21966 & 987 & 44.9 (42.2 to 47.8 ) & 20.7 (19.4 to 22.0$)$ & 251 & 11.4 (10.1 to 12.9 \\
\hline
\end{tabular}

$\mathrm{CMR}=$ crude mortality rate; SMR: standardised mortality ratio; $\mathrm{x}=$ suppressed due to cell size $<10$.

* Drug misuse or accidental drug poisoning (see appendix table A4).

†Per 1000 person years.

$\ddagger$ See appendix table A5 for ICD-9/ICD-10 codes used to identify concurrent chronic conditions.

$\S$ Any indication of non-opioid and non-alcohol drug use, poisoning (accidental or intentional), or substance use counselling or rehabilitation.

I Any indication of depression, anxiety, psychotic illness, personality disorders, attention-deficit/hyperactivity disorders, or bipolar disorders.

** Non-cancer chronic pain.

††Data linkage to provincial HIV and hepatitis $\mathrm{C}$ virus testing databases not available. 
Table 3| Relative risks of mortality off opioid agonist treatment (OAT) versus on OAT (reference), according to key periods of opioid overdose public health emergency in British Columbia, Canada, 1 January 1996 to 30 September 2018

\begin{tabular}{|c|c|c|}
\hline \multirow[b]{2}{*}{ Period } & \multicolumn{2}{|c|}{ Risk ratio $(95 \% \mathrm{Cl})$} \\
\hline & Unadjusted & Adjusted $^{*}$ \\
\hline Overall & 2.2 (2.1 to 2.4$)$ & 2.5 (2.1 to 2.9$)$ \\
\hline Period 1: before first death involving fentanyl $\ddagger$ & $1.8(1.7$ to 2.0$)$ & 2.1 (1.8 to 2.4$)$ \\
\hline Period 2: after first death involving fentanyl§ & 2.4 (2.2 to 2.7$)$ & $2.6(2.1$ to 3.2$)$ \\
\hline Period 3: after public health emergency declaration & 3.1 (2.8 to 3.4$)$ & 3.4 (2.8 to 4.3$)$ \\
\hline
\end{tabular}

Risk ratios compare crude mortality rate off OAT versus on OAT.

* Adjusted for age, sex, medication type (buprenorphine/naloxone only, methadone only), OAT period ( $\leq 4$ weeks or $>4$ weeks since starting or stopping OAT).

†Entire follow-up period (1 January 1996 to 30 September 2018).

$\ddagger$ Beginning of follow-up (1 January 1996) to day before first fentanyl related death in British Columbia (31 March 2012).

$\S$ Date of first fentanyl related death in British Columbia (1 April 2012) to day before public health emergency declaration (13 April 2016)

I Date of emergency declaration (14 April 2016) to end of follow-up (30 September 2018). 


\section{Figures}
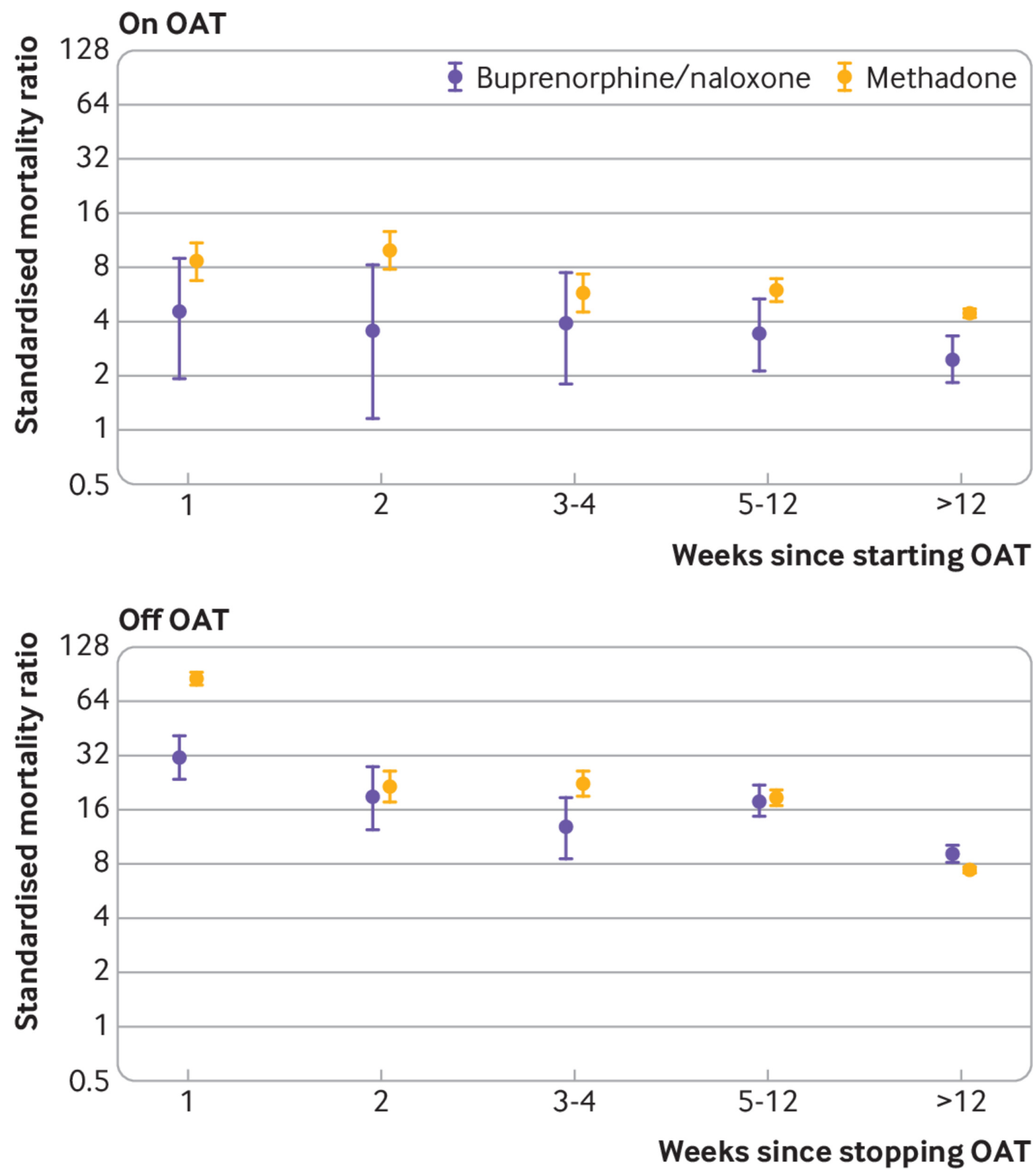

Fig 1 All cause mortality by time interval on and off opioid agonist treatment (OAT). British Columbia, 1 January 1996 to 30 September 2018 


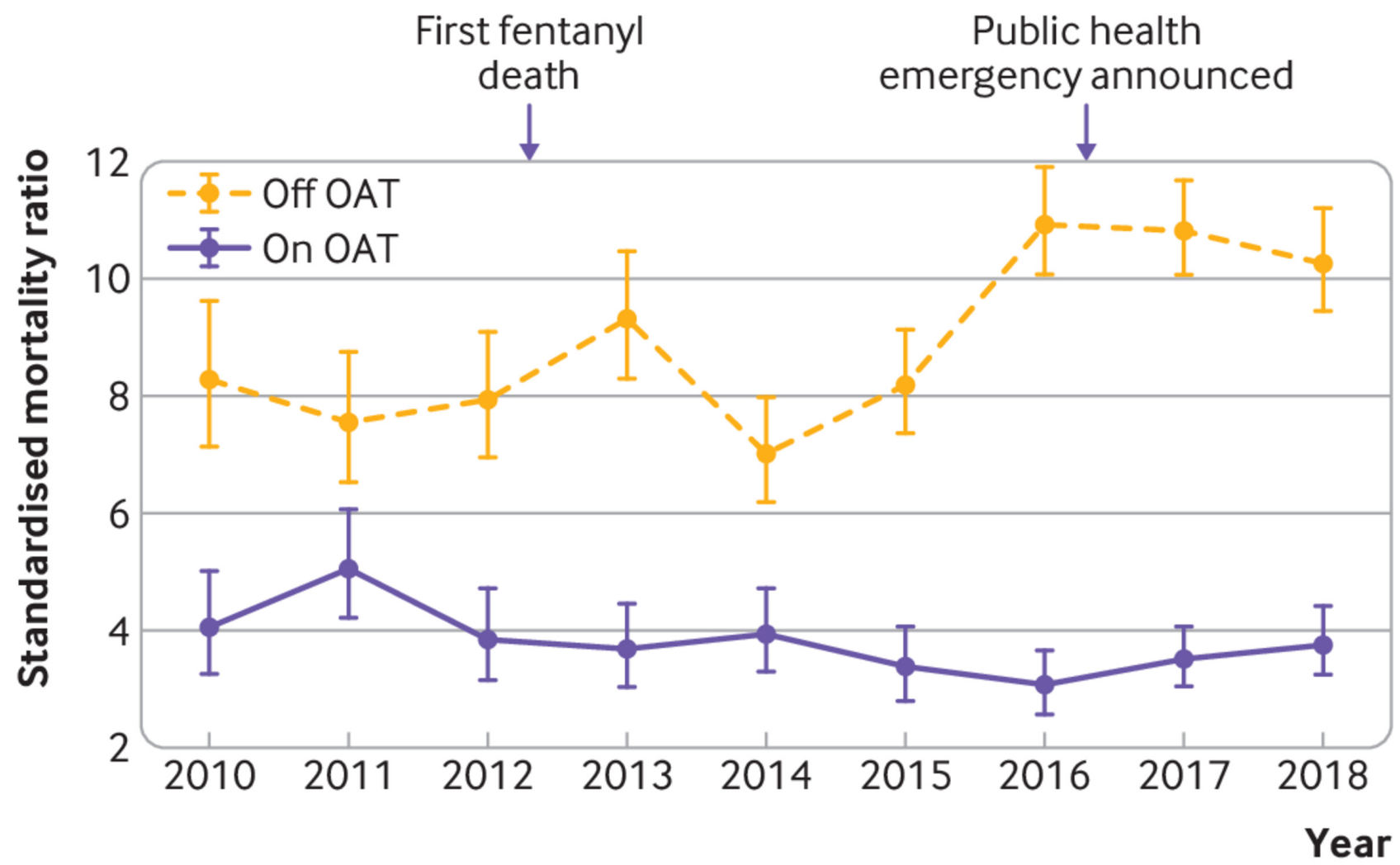

Fig 2 All cause mortality on and off opioid agonist treatment (OAT) in presence of fentanyl. British Columbia, 1 January 1996 to 30 September 2018 

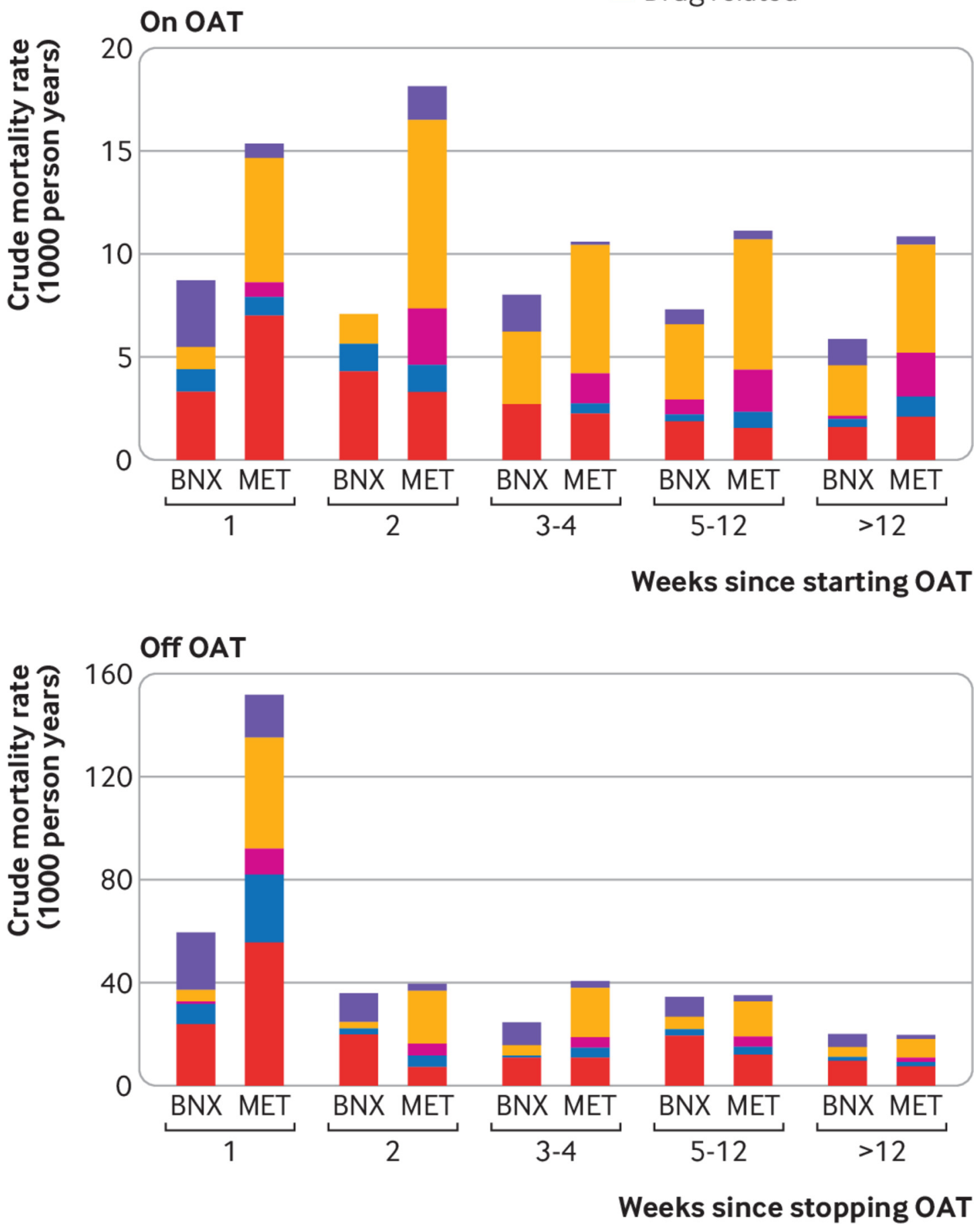

Fig 3 Cause specific mortality by time interval on and off opioid agonist treatment (OAT). British Columbia, 1 January 1996 to 30 September 2018. BNX=buprenorphine/naloxone; MET=methadone 